\title{
INDONESIAN VEHICLES NUMBER PLATES RECOGNITION SYSTEM USING MULTI LAYER PERCEPTRON NEURAL NETWORK AND CONNECTED COMPONENT LABELLING
}

\author{
Andre Sitompul ${ }^{\# 1}$, Mahmud Dwi Sulistiyo ${ }^{* 2}$, Bedy Purnama \#3 \\ \# School of Engineering, Telkom University \\ Jalan Telekomunikasi no.1, Kabupaten Bandung, Jawa Barat, Indonesia \\ ${ }^{1}$ andrede768@gmail.com, ${ }^{2}$ mahmuddwis@gmail.com, ${ }^{3}$ bedy.purnama@gmail.com
}

\begin{abstract}
In recent years, the amount of vehicle in Indonesia has been increasing rapidly. This surely, if it is conducted conventionally, challenges the upholder in recognizing and detecting the lawbreakers vehicle. The objective of this research aims to create the system which can automatically recognize vehicles number plates. This is also expected to be able to assist the upholder to take an action against the lawbreaker. The method used are sliding concentric windows and connected component for detecting and segmenting each of character on vehicles number plates. Further, multi-layer perceptron neural network classification model is used to identify each of character on it.

The system has been tested using variety of vehicles number plate images and succesfully recognize 180 of 224 characters images $(80.35 \%)$. Based on the computation of each character, the accuracy of the system, throughout tested vehicles number plate images, can reach $95.69 \%$ (1509 of 1577 characters can be identified).The tested system has shown prospective results, thus the technique used on this research can be implemented through vehicles number plate recognition system in Indonesia.
\end{abstract}

\section{INTRODUCTION}

The transportation system in the country is the important things to be maintained and enhanced. If the system is not sufficient, it will affect the problem in controlling and evaluating the lawbreakers vehicle. It might be caused by an inefficient system used for evaluating the lawbreaker. For instance, filling out vehicle identities and type of offenses may take too long time and complicated procedures. As the result, it is required the system which can recognize automatically the vehicle identity of lawbreaker. Recently, the technology that developes automatically the identification system has important roles for data-entry because when it comes to various data, It requires new effective ways of data-entry to electronic media. One of the ways is using Optical Character Recognition (OCR) technology.

Some methods had been used in the previous research in terms of identifying vehicles number plate. Regarding to [1] and [2], there are some factors influenced the vehicles number plate recognition process. For instance, the bad lighting and faded image gained during image acquisition processes. Moreover, the system found the problem when distinguishing similar character of letters and of number. For instance, letter B and number 8, letter A and number 4, letter I and number 1, letter Z and number 2, letter $\mathrm{G}$ and number 6 .

In this research, the use of connected component labelling method by improving some methods, used in identifying vehicles number plate, is expected to overcome the problem. The following are the improvement conducted through the system: First improvement is segmenting vehicles number plate into three segments and grouping the existed characters based on their own segment. Concerning to Indonesian Police standard system, the first part on vehicles number plate is the code of area (letter), the second part represents police number (Number), and the last part shows the last series of area.

The grouping of each character of vehicles number plate is conducted by measuring the space of character one another. If the space of previous character and next character exceeds the limit, the next character will involve in the different segment. By grouping each character based on its segment, the gained character on image process can be distinguished by letter or number. This affects the emergence of second improvement processed on multilayer perceptron neural network. It is conducted by creating two networks including identifying the character of letter and of number. This paper consists of several chapters as follows, and the next chapter will discuss the previous researches. Chapter III and VI describe the used method. Chapter $\mathrm{V}$ and chapter VI explain system planing and system implementation of vehicles number plate recognition. The last, Chapter VII and Chapter VIII discuss the tested results and conclusion and suggestions

\section{PREVIOUS RESEARCH}

On the previous research, Algorithm for vehicles number plate recognition had been developed generally by 4 steps [3] [4] [2] such as pre-procesing-image, location detection of vehicles number plate images, character segmentation, and character 
recognition of vehicles number plates.

There were some methods used in the previous research for detecting the location of vehicles number plates. One was the edge detection method using sobel operator which was used by [4] to identify vehicles number plates in Malaysia. The objective was to create the vehicles number plate recognition system in Malaysia which could extract the location by unlimited image acquisition process, such as the space and large angle between the camera and the car, and image quality. By 150 image processed, the research had succesfuly gained $92.1 \%$ in identifying vehicles number plate location, $90,5 \%$ separating the number plate from the real image, 93,2 processing identified characters. However, [1] described that this was inapropriate method to be used in equal color of car and of number plate. It was caused by insufficient light changing on number plates resulting in unprocessed edge detection. Moreover, based on [2] and [3], edge detection method would produce improper level of accuracy. It happened when it was adapted to the low-quality images such a motion blur and excessive noise level [6] had conducted the different approach for detecting and segmenting the image location of vehicles number plates using sliding concentric windows method. Regarding to the research conducted in Yunani, this method had successfully detected and segmented about 1287 of 1334 vehicles number plates $(96,5 \%)$ on the image used as the input. To recognize the character of them, He had used Probabilistic Neural Network (PNN). By implementing this model, this research had reached $89,1 \%$. The average time needed to recognize vehicles number plates was about 276 millisecond. [7] was conducting the similar research in Korea. The research consisted of 3 steps including detecting the real vehicles number plates using SCW method, verifying green and white color based on HIS color model, and segmenting by histogram. About 82,5\% from Around 40 data processed, had been segmented correctly.

Furthermore, there were some methods used for detecting and identifying the location of vehicles number plates. Those were the use of Hough Transformation to detect the line which was assumed as the real number plates [8], of fuzzy logic which required some rules and knowledge of number plate to show the location, and of Pulse Coupled Neural Network (PCNN) to identify the character [9]. Besides, there were the use of data kd-tree structure and approximate nearest neighbor which was used to obtain the pixels of the image of real vehicles number plates [10].

\section{CONNECTED COMPONENT LABELLING}

Connected component labelling is an algorithm which is applied based on graph theory and can be used as the segmenting method of the the image elements. Connected component labelling is well-known as connected component analysis. This is because, after labeling process, the objects will be analyzed to get the proper object regarding to the features which has been defined. Labelling process is commonly conducted to mark the objects connected to the binary image.

Labeling process of the binary image is conducted by attaching the similar label on the adjoining elements. For instance, black and adjoining element will be labelled 0 , while white and adjoining element will be labelled 1 . On the binary image, the black element is called nodes, while the paired black adjoining element is called edges. Controlling the adjoining relationship of each element (pixel) can be carried out by two ways either 4-adjacent or 8-adjacent. Both of ways are checking the labels on the adjoining elements.

\section{SLIDING CONCENTRIC WINDOWS}

Sliding Concentric Windows is the method used to show local irregularity on image. This method works by forming two different size of windows, however it has similar center. Both of windows will control randomly each of image pixel and will compare to statistic value (average/deviation standard) gained from pixel values on its window. This method can be used to classify whether the checked pixels (the center) involves on region of interest (ROI) or not. This method is conducted by the the terms as follows: for instance, $\mathrm{x}$ and $\mathrm{y}$ is the pixel coordinate which will be checked (The center), W1 wide $\mathrm{x} 1$ and $\mathrm{y} 1$ and W2 by wide $\mathrm{x} 2$ and high y2are the used windows, and $\mathrm{A}$ is the source image. Thus, the pixel values on $\mathrm{x}$ and $\mathrm{y}$ coordinate of target image is image A_2 which will be set to be 0 (not ROI) or 1 (ROI) in accordance with the condition as follows [6]:

$$
\text { in } A_{1}(x, y) \rightarrow \begin{cases}A_{2}(x, y)=0, & \text { jika } \frac{M_{w 2}}{M_{w 1}} \leq T \\ A_{2}(x, y)=1, & \text { jika } \frac{M_{w 2}}{M_{w 1}}>T\end{cases}
$$


$\mathrm{M}$ is the value measurement of pixel statistics in windows (W), and $\mathrm{T}$ is the threshold which has been determined. Both of those windows will entirely check all of image pixels. Windows size determination W1 and W2 can be arranged as needed. However, for threshold values, there is no exact size which can be used for obtaining optimal results. So, it can be gained by using trial and error methods. The illustration of sliding concentric windows can be seen at 1 (A) and 1 (B)

[A]

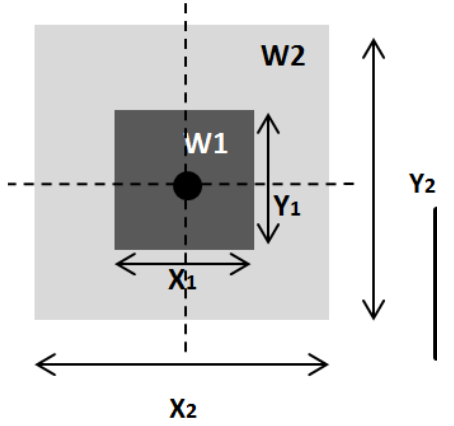

2

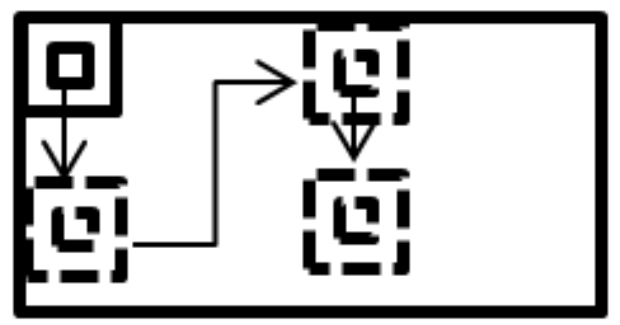

[B]

Fig. 1. (A) Concentric Windows (B)Scanning process conducted by sliding concentric windows

\section{SYSTEM DESIGN}

\section{A. Data Analysis}

The raw data processed as the sample on this research is the 2736 x 2736 pixel image which is obtained using Olympus XZ-1 digital camera with 10 MP sensor resolution. The size selection of image angel is equally processed that the vehicle location will be easly positioned when it is being captured. The variety space between the camera and the vehicle during image capturing process starts from $1 \mathrm{~m}$ to $2 \mathrm{~m}$ with the height $1.5 \mathrm{~m} 1.7 \mathrm{~m}$.

The collected sample are 310 images. Those will be categorized by 5 different data sets and each will be cropped manually to get vehicles number plates on the image. The first data set will be processed as the training data and the rest will be the test data. Table 1 shows the composition of each data set used as the sample in this research

\section{B. general overview of the system}

After the process of acquisition and categorization of image to the different data sets, the training data set will be primarily processed to have each of images character. There are mainly 4 steps to detect and recognize vehicles number plate character such as the pre-processing image, character segmentation, feature extraction, and character identification. The extracted feature will involve in neural network as the input processed to identify vehicles number plate character. The circulation process of the system can be generally seen in picture 2 .

\section{VEHICLES NUMBER PLATE RECOGNITION SYSTEM}

\section{A. Pre-Processing Image}

Pre-processing image is the first step carried on after image reading process by system. This step involves the resizing process of vehicles number plate image into 252 × 72 pixel using Lanczos method. This aims to make the similar image size which will be processed by the system. The next process is converting the image into the graysclae image. Then, it will be inverted and processed using sliding concentric windows method to certainly make the pixels on the image of vehicles number plate in Regions of Interest (ROI). The pixel in ROI will be given 1 and those out of ROI will get 2. The size of two windows, used as the parameter on images sliding concentric windows process, is based on aspect ratio (wide:height) from the character of Indonesian vehicles number plates. The character has generally comparative ratio $1: 2$, therefore the high value (Y) which was selected for each window is twice bigger than the wide value (X). For instance, if $X \_1=2$, so $Y \_1=4$ and if $X \_2=8$, so Y_2=16. Determining $X$ and $Y$ parameter for $\mathrm{W}_{-} 1$ and $\mathrm{W} \_2$ is freely conducted in accordance with the term of aspect ratio. Moreover, threshold value determining is processed using trial and error method to obtain the optimal results because there is no exact size to be used to determine threshold value [6]. After the SCW process of the image, the result will be binary image. The SCW adjustment for each data set on the system can be showed in table 2

Furthermore, after the process of sliding concentric windows method, the output will be the binary image which has 0 or 1. The next process is the connected component labelling method. This method is one of the used techniques for labelling 
TABLE I

SET DATA COMPOSITION

\begin{tabular}{|c|c|c|c|c|}
\hline Sample & Space $(\mathrm{m})$ & Height (m) & Description & Total of Images \\
\hline Dataset-1 & $1-2$ & $1.5-1.7$ & $\begin{array}{c}\text { Parking area of } \\
\text { Telkom University } \\
\text { (School of } \\
\text { Engineering), Morning } \\
\text { (10 a.m. } 11 \text { a.m.) }\end{array}$ & 86 \\
\hline Dataset-2 & $1-2$ & $1.5-1.7$ & $\begin{array}{c}\text { Parking area of } \\
\text { Telkom University } \\
\text { (School of } \\
\text { Engineering), Morning } \\
\text { (10 a.m. } 11 \text { a.m.) }\end{array}$ & 75 \\
\hline Dataset-3 & $1-2$ & $1.5-1.7$ & $\begin{array}{c}\text { Parking area of } \\
\text { Bandung Indah Plaza, } \\
\text { Afternoon (1 p.m. } 3 \\
\text { p.m.) }\end{array}$ & 48 \\
\hline Dataset-4 & $1-2$ & $1.5-1.7$ & $\begin{array}{c}\text { Parking area of } \\
\text { Bandung Institute of } \\
\text { Technology, Afternoon } \\
\text { (1 p.m. } 3 \text { p.m.) }\end{array}$ & 48 \\
\hline Dataset-5 & $1-2$ & $1.5-1.7$ & $\begin{array}{l}\text { Parking area of Husein } \\
\text { Sastranegara } \\
\text { International Airport, } \\
\text { morning (7 a.m. } 8 \\
\text { a.m.) }\end{array}$ & 45 \\
\hline Total of Images & & & & 310 \\
\hline
\end{tabular}
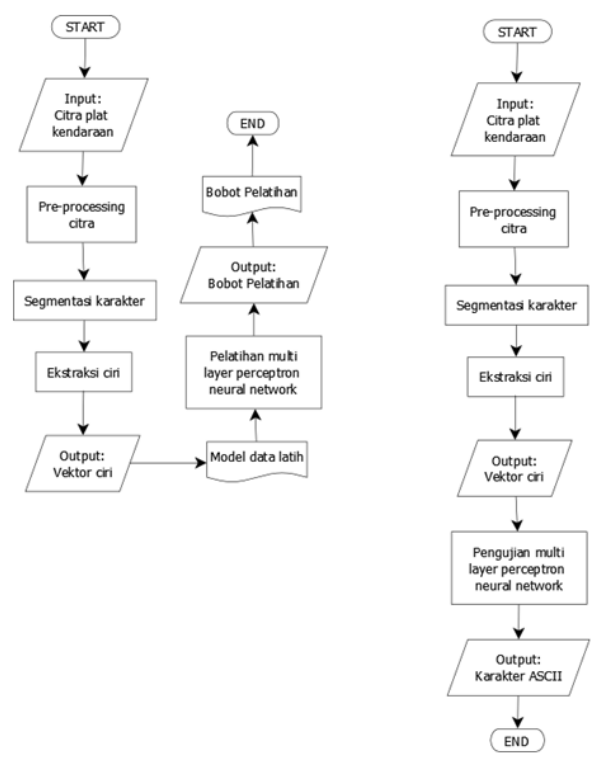

Fig. 2. General Flowchart System

TABLE II

THE ADJUSTMENT OF SCW ON EACH DATASET

\begin{tabular}{|c|c|c|}
\hline Dataset & Window size determination & Threshold value \\
\hline Dataset- 1 & $\mathrm{X} 1=2 ; \mathrm{Y} 1=4 ; \mathrm{X} 2=8 ; \mathrm{Y} 2=16$ & 0,985 \\
\hline Dataset-2 & $\mathrm{X} 1=2 ; \mathrm{Y} 1=4 ; \mathrm{X} 2=8 ; \mathrm{Y} 2=16$ & 0,985 \\
\hline Dataset-3 & $\mathrm{X} 1=2 ; \mathrm{Y} 1=4 ; \mathrm{X} 2=8 ; \mathrm{Y} 2=16$ & 0,985 \\
\hline Dataset-4 & $\mathrm{X} 1=2 ; \mathrm{Y} 1=4 ; \mathrm{X} 2=8 ; \mathrm{Y} 2=16$ & 0,995 \\
\hline Dataset-5 & $\mathrm{X} 1=2 ; \mathrm{Y} 1=4 ; \mathrm{X} 2=8 ; \mathrm{Y} 2=16$ & 0,995 \\
\hline
\end{tabular}

the areas of image which has interconnected pixels. By using this method, the areas involved in ROI will be attached by the 
different label to distinct one another. The labelling process is needed to analyze the ROIs area so those which has no character of vehicles number plate will be deleted from the image. Picture 3 presents the result of pre-processing on sample image as the input for the system.

[A]

$[\mathrm{C}]$
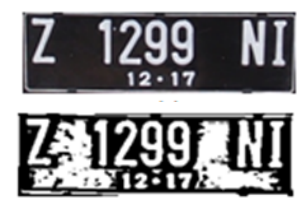

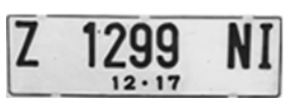

Z 1299 III

Fig. 3. (A) input (B)resized and converted image into grayscale (C)resized and converted image into grayscale (D) result after labelling and analysing process

However, the procedure can not ensure the result of character detection to vehicles number plate which has non-white characters and non-black background. Thus, if there is no detected character on image, the system will re-read, resize, and re-convert the input. Yet, the next process shows that the image is not inverted. Picture 4 presents the sample of pre-processing to vehicles number plate by yellow character and black background.

[A]
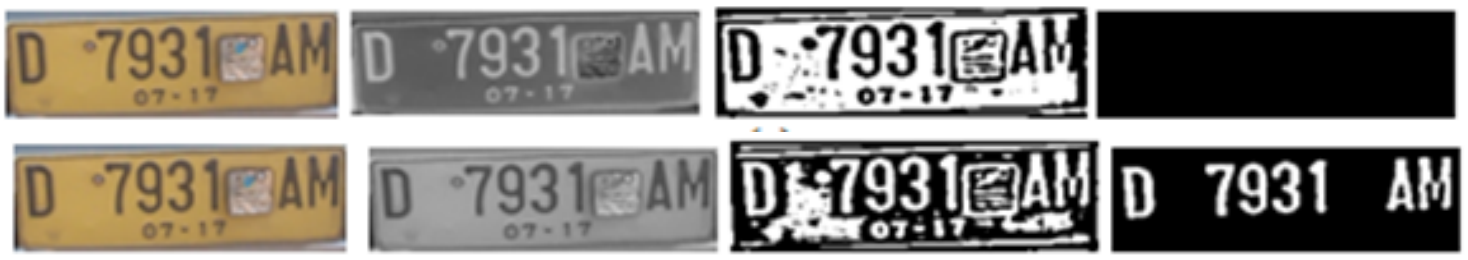

[B]
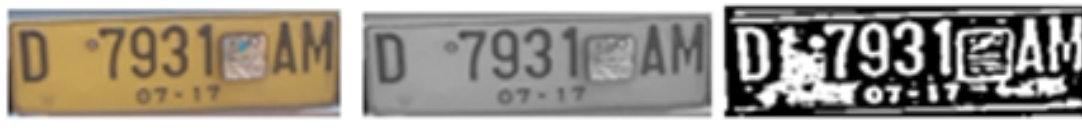

Fig. 4. (A) pre-processing result with inversion (B)pre-processing result without inversion

After the all-area labelling process, the image will be analyzed to gain the areas which is the character of vehicles number plates. The image remains the character of vehicles number plate by the features as follows:

1) $70^{\circ} \leq$ Orientation $\leq 140^{\circ}$

2) $38 \leq$ Height $\leq 60$ (pixel)

3) Wide area $\geq 100$ (pixel)

If the labelled area can not require those terms, it will not be adjusted as the character and will be deleted from the image. Therefore the output is merely the character on vehicles number plate.

\section{B. Character Segmentation}

The binary image made from preprocessing will be segmented to get each character of vehicles number plate. This step shows that the coordinate of each produced character will be saved for the next process. The future character segmentation process is highly influenced by the preprocessing succeed system. If the preprocessing identifies the undetected character by the system, it will fail, in segmentation process, to be segmented and will affect to the identification process of character of the next step. There has been a variety of character size from segmentation process. Therefore, the size of each character needs to be equated. It is carried on so the next extraction process of the future step will be held easily. The size of each character will resize into 20 x 40 pixel using Lanczos method. After the normalization process, each of them will be checked to be defined either as a letter or number. This process is managed by measuring the space of each character based on their own coordinate on the previous image. Concerning to Indonesian Police Standard System, the first part of vehicles number plate shows the code of region (letter), the second part remains police number (number), and the last part is the last serial of region (letter). Thus, the first character detected on segmentation process will be automatically defined as the letter. The rest of them is defined regarding to the space of one and another previous character. The calculation process of the space among the characters is carried on by reducing the space between the checked left-side pixel coordinate and the previous right-side pixel coordinate. If the calculation results in 15 pixel, the character lies on the similar segment with the previous character. It means that the letter/number is equal with the previous character. However, if the calculation is $i 15$ pixel, the character will be in the different segment so the type of them is different from the previous one. The number will be labelled by 1 while the letter by 2 . Picture 5 serves the result of segmentation process and defining the type of each caracter.

\section{Feature Extraction}

Before the character is on the multi-layer perceptron neural network as the input, the feature will be firstly extracted. It affects less input so there will be faster training and testing process of neural network. The feature of each character of raw 


\section{Z 1299 III

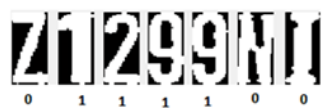

Fig. 5. The result of segmentation process and character defining

data will be extracted and saved as the training data used by neural network to train its network. While those from the tested image, will involve to neural network as the test data. Each of them will randomly go through neural network to be identified. The first extraction process of each character is thinning process. After this process, the $20 \times 40$ pixel image will be divided into 32 areas which consisted of 5 x 5 pixel ( 5 rows, 5 colums). Each of them will be represented to the average value of pixel which values 1 to the blok $5 \times 5$.

\section{Character Identification}

Identification character process or character recognizing is the last step in recognizing the character on vehicles number plates. Character recognizing is processed using artificial neural networks with multi-layer perceptron.

Through this process, two phases will be carried out to the artificial neural network model before recognizing each character as the input. The first phase is training phase which works for gaining optimal configuration network using processed training data. Due to that, when the network is being tested, the created network can adjust on input and output mapping. The second phase goes to the phase which identifies the character as the test data. This paper presents two artificial neural networks to be used. The first network works to train and recognize the data which involve the character while the second one carries on the letter. Each network will be created using back propagation learning method. The architecture of them consists of one input layer, one hidden layer, and one ooutput layer. The design of them can be seen in picture 6 and 7. More information can be described as follows:

1) Input layer of each network consists of 32 neurons started from 1st input to 32th input. Each of them is the value from the extraction process of character feature gained from the previous process.

2) Hidden layer of the first network consists of $n$ neuron, where $n$ values $=30,35,40,45$, and 50 while the other of second layer comes to $\mathrm{n}$ neuron with $\mathrm{n}$ values $=45,60,75,90,120$, and 130 .

3) Output layer of the first network consists of 10 neurons started from output 1 to output 10 while in the second network is started from 1 st output to 26 th output.

4) $x 1$ to $x 32$ is the neuron used on both of input layer networks.

5) On the first network, y1 to y10 is the neuron used in the output layer. Whereas, in the second network, the neuron used is $\mathrm{y} 1$ to $\mathrm{y} 26$.

6) b1 is the bias value used in hidden layer and b2 output layer.

7) w1ij is the connection load between 1 st neuron on input layer and $\mathrm{j}$ neuron on hidden layer. Furthermore, w2kl is the connection load between $\mathrm{k}$ neuron on hidden layer and 1 st neuron on output layer.

8) $\mathrm{w} 10 \mathrm{j}$ is the bias load for $\mathrm{j}$ neuron on hidden layer and w20k is for $\mathrm{k}$ neuron on output layer

After the process of defining network architecture, the next step is network training process. Those networks will be trained using the collected train data. Each of character has 5 pattern, for instance, 1 consists of 5 pattern, A has 5 pattern, and the simillar thing of other characters. Therefore, the total training data are 180pattern.

The testing network process is merely carried on using feedforward method. The value of extarction feature from the previous step will be the identified input. Moreover, those input values will be served to hidden layer and output value of hidden layer, counted and re-served to output layer. This results in numerical value which is the output of output layer. The calculation of hidden layers output value is used using activation function.

The output value will be firstly analyzed before comparing to the output vector. The highest output of matrix will be changed into 1 , then those in which values are less than 1 will be 0 They will be compared to output vector to obtain proper target value (character).

\section{TEST RESULTS}

Vehicles number plate recognition system is created to be tested using two scenarios to multi-layer perceptron neural network. The first scenario has only one network which can recognize letter and number on vehicles number plates. The second scenario process two networks including 2.1 and 2.2. Network 2.1 is used to identify the numbers while 2.2 is used to recognize letters. The network architecture used to the first scenario are as follows: input layer consists of 32 neuron, output layer 36 neuron, and the total hidden neuron are 50,60,80,100,120, and 130. The left scenario comes to the network showed in picture 6 and 


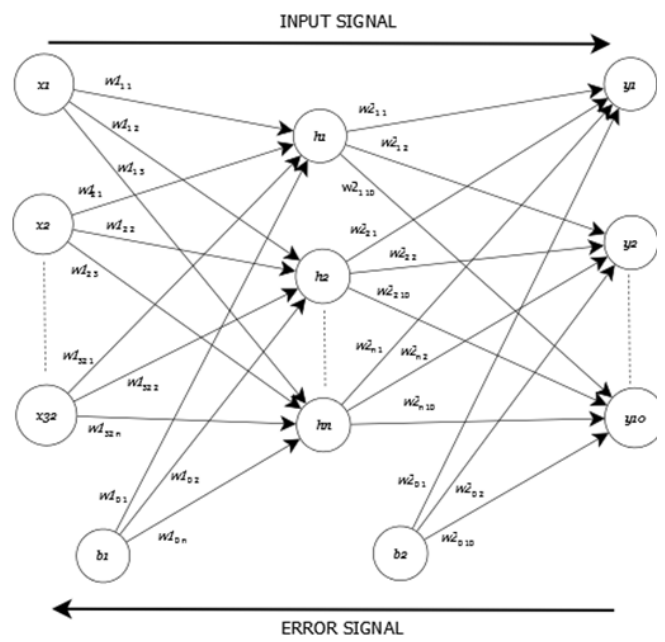

Fig. 6. The first network architecture used to recognize number (0-9)

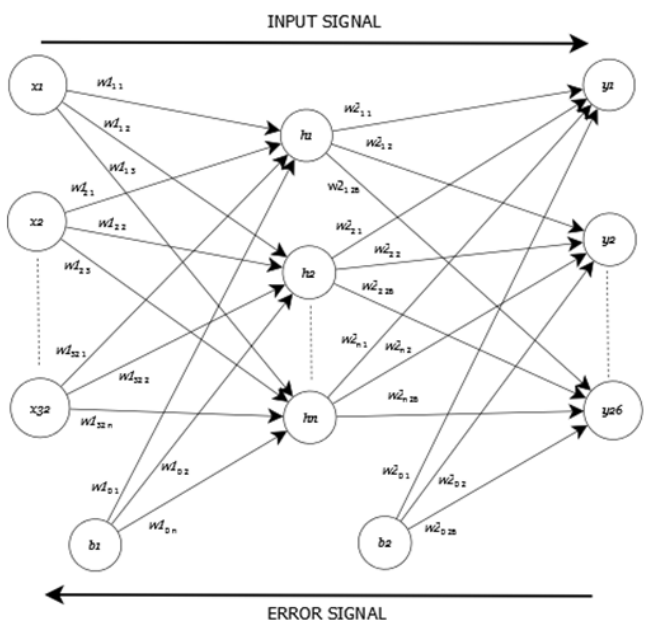

Fig. 7. The second network architecture used to recognize letter (A-Z)

7. The use of both scenarios aims at comparing the system performance using one-network system created by two-network system in recognizing the characters on number plate. The tested results of them can be seen in table 3 and 4 . From those tables, system performance can be measured concerning to the system ability in recognizing the whole number plate. Hereby the formula used to measure the system performance:

$$
\begin{aligned}
\text { SystemPerformance } & =\frac{\text { Totalidentifiedcharacters }}{\text { Totalcharacters }} \\
\text { SystemPerformance } & =\frac{\text { TotalidentifiedVehicle }}{\text { Totalvehicle'snumbersplate }}
\end{aligned}
$$

The following are the system performance gained from each scenario:

Scenario A

1) System performance in recognizing character:

$$
\frac{1478}{1577}=93,72 \%
$$

2) System performance in recognizing vehicles number plates:

$$
\frac{143}{224}=63,84 s \%
$$

Scenario B 
1) System performance in recognizing character:

$$
\frac{1509}{1577}=95,69 \%
$$

2) System performance in recognizing vehicles number plates:

$$
\frac{180}{224}=80,35 \%
$$

TABLE III

TESTED RESULTS SCENARIO A (1NETWORK)

\begin{tabular}{|c|c|c|c|c|}
\hline Test data & $\begin{array}{c}\text { Total identified } \\
\text { characters }\end{array}$ & Total characters & $\begin{array}{c}\text { Total identified } \\
\text { vehicles number } \\
\text { plates }\end{array}$ & $\begin{array}{c}\text { Total vehicles } \\
\text { number plates }\end{array}$ \\
\hline Dataset 2 & 502 & 527 & 52 & 75 \\
\hline Dataset 3 & 323 & 347 & 30 & 48 \\
\hline Dataset 4 & 370 & 395 & 35 & 0.5610 \\
\hline Dataset 5 & 283 & 308 & 26 & 0.5821 \\
\hline Total & 1478 & 1577 & 143 & 0.5634 \\
\hline
\end{tabular}

TABLE IV

TESTED RESULTS SCENARIO B (2NETWORKS)

\begin{tabular}{|c|c|c|c|c|}
\hline Test data & $\begin{array}{c}\text { Total identified } \\
\text { characters }\end{array}$ & Total characters & $\begin{array}{c}\text { Total identified } \\
\text { vehicles number } \\
\text { plates }\end{array}$ & $\begin{array}{c}\text { Total vehicles } \\
\text { number plates }\end{array}$ \\
\hline Dataset 2 & 502 & 527 & 52 & 75 \\
\hline Dataset 3 & 329 & 347 & 38 & 48 \\
\hline Dataset 4 & 380 & 395 & 48 & 0.5610 \\
\hline Dataset 5 & 297 & 308 & 37 & 0.5555 \\
\hline Total & 1509 & 1577 & 180 & 0.5400 \\
0.5406 & \\
\hline
\end{tabular}

\section{CONCLUSION AND SUGGESTIONS}

Regarding to the analysis and test conducted, there are some conclusions including connected component labelling dan multi layer perceptron neural network method with backpropagation learning algorithm which present the prospective results in recognizing vehicles number plate in Indonesia. The result shows that the system can be used by police officer so their recognition process on vehicles number plate can be easily conducted. Furthermore, the use of two multi-layer perceptron neural network in recognizing letter and number on vehicles number plate serves the faster and better results in identifying the number plate comparing to one multi-layer perceptron neural network system. It is because of the categorization of each character based on their segment to carry out better performance differentiating number and letter. The suggestions for the future research are implementing smartphone-based recognition system for vehicles number plates to get the real-time of image processing and its identification process. Besides, the detection process of its location on image can be developed so it may not be cut manually to get part of it on the image. The future research in integrating character value of ASCII, gained from vehicles number plate processing on polices database system, can be easily conducted that functions to process the information related to checked vehicle. To improve the system performance in recognizing vehicles number plate, there will be the future research focused on determining total of pattern for each character on train data. It ables to identify more pattern of character on number plate. However, the period of train data model must be the important thing to be used to train the network. Moreover, the future research is conducted to determine better segmentation method to separate the character of number plate and can be conducted to improve system performances.

\section{REFERENCES}

[1] B. D. Acosta, EXPERIMENTS IN IMAGE SEGMENTATION FOR AUTOMATIC US LICENSE PLATE RECOGNITION, Jun. 2004.

[2] F. Odone, Experiments on a License Plate Recognition System, 2007.

[3] V. Mirashi, J. Parab, M. Shirvoikar, R. Kudaskar, and S. Borkar, License Plate Detection and Segmentation for Goan Vehicles, vol. 2, no. 2, Feb. 2013.

[4] O. Khalifa, S. Khan, R. Islam, and A. Suleiman, Malaysian Vehicle License Plate Recognition, vol. 4, 2007.

[5] G. T. Shrivakshan and C. Chandrasekar, A Comparison of Various Edge Detection Techniques Used in Image Processing, IJCSI, vol. 9, no. 5, Sep. 2012.

[6] C. N. E. Anagnostopoulos, I. E. Anagnostopoulos, V. Loumos, and E. Kayafas, A License Plate-Recognition Algorithm for Intelligent Transportation System Applications, IEEE, vol. 7, no. 3, pp. 377392, Sep. 2006. http://www.news-medical.net/health/What-is-a-Brain-Tumor-(Indonesian).aspx

[7] K. Deb, H.-U. Chae, and K.-H. Jo, Vehicle License Plate Detection Method Based on Sliding Concentric Windows and Histogram, J. Comput., vol. 4, no. 8, Aug. 2009. 
[8] SV. Kamat and S. Ganesan, An Efficient Implementation of the Hough Transform for Detecting Vehicle License Plates using DSPs, IEEE, pp. 5859, 1995.

[9] Q. Gao, X. Wang, and G. Xie, License Plate Recognition Based on Prior Knowledge, IEEE, pp. 29642968, 2007

[10] J. Cano and J.-C. P.-C. Cortez, Vehicle License Plate Segmentation in Natural Images, vol. 2652, pp. 142149, 2003.

[11] M. B. Dillencourt, H. Samet, and M. Tamminen, A General Approach to Connected-Component Labeling for Arbitrary Image Representations, 1992. 